\title{
NEUROTICISMO Y TRASTORNOS DE PERSONALIDAD
}

\section{Vicente Pelechano \\ Alberto Pastor}

Universidad de La Laguna (Tenerife) ${ }^{1}$

\section{RESUMEN}

Una muestra de 793 adultos (18-64 años de edad, 62,9\% mujeres) cumplimentaron el IPDE de Loranger. 459 de ellos además, cumplimentaron la escala de neuroticismo de Eysenck (EPI). Los coeficientes de correlación corregidos por atenuación muestran que neuroticismo resulta estrechamente vinculado a los trastomos especificos de personalidad que recoge ta CIE-10 excepto el esquizoide y el disocial. Los ítems de las escalas criteriales de trastomos responsables de esta relación se refieren a componentes de inseguridad social y hostilidad, que se encuentran repartidos en prácticamente todas las escalas. Se presentan resultados sobre el poder diferencial que poseen edad, género, nivel profesional y estado civil. Los resultados demuestran que la dimensión de neuroticismo es muy importante a la hora de encontrar anclajes y correlatos de personalidad de los trastomos de personalidad. Otras dimensiones propuestas por el modelo de $H$. J. Eysenck son importantes para trastomos específicos (introversión para esquizoide y probablemente psicoticismo para disocial). Se ofrecen datos para una psicología diferencial de los trastomos de personalidad.

Palabras clave: TRASTORNOS DE PERSONALIDAD, TRASTORNOS DE PERSONALIDAD Y NEUROTICISMO, TRASTORNOS DE PERSONALIDAD Y DIMENSIONES DE PERSONALIDAD, PSICOLOGÍA DIFERENCLAL DE LOS TRASTORNOS DE PERSONALDAD.

1. La correspondencla sobre este trabajo puede dirigirse al primer autor: vpelecha 


\section{SUMMARY}

793 adults (18-64 years, 92,9\% women) fulfilled the IPDE questionnaire by Loranger on personality disorders (ICD-10 classification). A subsample of 459 fulfilled the $\mathrm{N}$-scale by Eysenck (EPI questionnaire). Correlation coefficients corrected by attenuation among neuroticism and personality disorders are high and very high except in the case of schizoid and dissocial personality disorders. The main responsibility of this result depends of items related to social insecurity, hostility and rigid adherence to social rules and norms which are present in the most part of the criterion scales. Results are also shown on the differential power of the age, gender, civil status and professional level. Results show that neuroticism is an important dimension to understand the most part of the specific personality disorders (ICD-10); introversion seems to be important for schizoid personality disorder and psychoticism for dissocial personality disorder. Substantially, these results show that personality dimensions are important determinants and/or correlates of personality disorders, in front of a part of the published literature. Results are offered on differential psychology of personality disorders.

KeY WORdS: PERSONALITY DISORDERS, PERSONALITY DISORDERS AND NEUROTICISM, PERSONALTTY DISORDERS AND PERSONALITY DIMENSIONS, DIFFERENTIAL PSYCHOLOGY OF PERSONALITY DISORDERS.

En el lúltimo tercio del siglo $X X$ se consolidaron como objeto de diagnóstico, tratamiento e investigación un grupo de problemas psicopatológicos de gravedad dispar aunque todos ellos perturbadores de la convivencia interpersonal que se identificaron como "trastornos de personalidad". Realmente no eran problemas nuevos sino que ya habían sido detectados, en su mayor parte, en la Grecia del siglo $V$ antes de Cristo $y$, de una $u$ otra manera, han estado perviviendo hasta nuestros días. Como grupo no tienen muchas cosas en común, aunque todos ellos son molestos, al menos, para las personas que viven con quienes los sufren (las personas que los sufren pueden tener problemas debido a ellos o no), poseen un cierto carácter ético, son resistentes a los tratamientos farmacológicos conocidos y el éxito que se tiene con las intervenciones psicológicas 
es, más bien, pobre. Se desconoce la base biológica de cada uno de ellos y han sufrido cambios en conceptuación y.clasificación en cada ocasión que se ha intentado estudiar y ordenar lo que de ellos se conoce hasta el punto, que en éste caso existen claramente diferencias entre los dos grandes sistemas clasificatorios de consenso, el DSM y la CIE (pese a los intentos realizados para su convergencia conceptual y diagnóstica). En cada versión de estos sistemas se han incluido un número distinto de entidades psicopatológicas $\theta$ incluso los descriptores de ellas se han modificado de versión en versión de los sistemas clasificatorios (incluso dentro de una misma opción, en el DSM-IV se encuentran diferencias entre la última versión de 1994 y la revisión de texto propuesta en el año 2000 (DSM-IV-TR)). Esta "modificabilidad" de unos trastornos que se suponen "inmodificables" llama la atención y es interpretada desde dos perspectivas radicalmente opuestas: o bien como una muestra de la relevancia del campo y de una copiosa actividad de investigación que promueve estos cambios (lo que es verdad en parte como se muestra en Miguel y Pelechano (2000)); o alternativamente, que existen problemas graves en la delimitación conceptual y operativa de los mismos (Deary et al.,1998) y por ello, se sugiere incluso su eliminación de los sistemas clasificatorios, en la medida en que muchos de los síntomas que se dan en esos problemas se encuentran recogidos asimismo en los trastomos "especfficos" (trastornos en el eje uno de los DSM). El caso es, sin embargo, que pese a las dificultades inherentes a su estudio, existen correlatos y predicciones distintas cuando las personas vienen aquejadas de un trastorno específico $y$, además, uno u otro trastomo de personalidad (Costello, 1996) en el sentido de que estos trastornos siempre representan un hándicap supletorio para las personas que los sufren hasta el punto de que su presencia disminuye al $50 \%$ la expectativa de éxito de ciertos trastomos como la depresión y la ansiedad; por otra parte, la experiencia demuestra que se obtienen efectos paradójicos cuando se aplican fármacos que son eficaces en los trastomos específicos (tal y como sucede con los antidepresivos en los trastomos límite de personalidad).

La idea básica de estos trastornos es que representan conjuntos de rasgos de personalidad con carécter claramente desadaptativo, 
difíciles cuando no imposibles de erradicar, hoy por hoy, y que conforman la vida de las personas de manera tan "natural" que resulta diffcil que estas personas piensen que necesitan ayuda (son ego-sintónicos). En los estudios publicados, curiosamente estos "trastornos" tienden a presentar relaciones más bien tenues con los rasgos estudiados por la psicologia de la personalidad. Y este desajuste resulta preocupante en la medida en que en ambos casos se utilizan "rasgos" como unidades básicas de análisis (en algunos modelos se sustituyen los rasgos por esquemas o guiones), rasgos que tienen su lugar de asentamiento en la psicología de la personalidad $y$, en ella han tenido un notable desarrollo. En la medida en que se pudiesen demostrar relaciones significativas, los trastornos de personalidad podrían beneficiarse del considerable volumen de conocimientos que posee la psicología de la personalidad, tanto por lo que se refiere a la estructura personal como a su dinámica ( $y$, desde ahí, al cambio terapéutico). Finalmente, habria que señalar que este vacío resulta tanto más inexplicable por cuanto que una de las tradiciones de mayor peso en la constitución y desarrollo de la psicologia de la personalidad es el pensamiento diferencial médico y clínico en general (Pelechano, 1993).

Existen muchas razones posibles acerca de esta separación entre personalidad y trastornos de personalidad y muchas de ellas han sido objeto de estudio específico. Se pueden agrupar en dos grupos conceptuales (téricos o metodológicos): o se trata de un problema de los predictores (de los propios rasgos de personalidad), o se trata de un problema del criterio (de los identificadores - marcadores de los trastornos de personalidad). Usualmente los clínicos defienden el primer bloque; los psicólogos básicos de la personalidad, el segundo.

Las criticas principales aducidas a la psicología de la personalidad son las siguientes: (a) no existe un paradigma estructural básico y aceptado por todos o la mayorla de científicos acerca de cuáles son los rasgos que forman la personalidad del ser humano adulto, de las relaciones entre ellos (estructura) y de su dinámica; (b) la mayor parte de las investigaciones sobre la estructura de la personalidad se ha preocupado de aislar factores que no son directamente re- 
levantes para la clínica ${ }^{2} y$, cuando se han aislado factores relevantes, no han sido validados de manera adecuada; (c) los rasgos aislados han estado excesivamente o muy cargados de teoría (como la teoría de $H$. J. Eysenck), o ausentes de ella (como sucede en el modelo de los cinco grandes y con base correlacional y no experimental sensu stricto), o con teorias débiles y de alcance muy reducido (como ha sucedido con la dependencia y la independencia de campo o el locus de control) o, con teorlas demasiado complejas para ser susceptibles de contrastaciones parciales fáciles de realizar (como ha sucedido con la teorla de R. B. Cattell) o, con elaboraciones alejadas de la necesaria flexibilidad y cercanía a los problemas relevantes que se encuentran presentes en la clínica psicológica $y$, finalmente (d) se requiere una lógica distinta a la multivariable con grandes números de personas para acercarse a los problemas reales de la clínica. A estas críticas añadirlamos que se han elaborado teorías de un solo nivel estructural de la personalidad, posiblemente demasiado rigidas y elementales para poder ser aplicadas en el mudo clínico y de relaciones interpersonales.

Ante estas críticas habría que decir que si bien es verdad que existen multiplicidad de teorlas y propuestas distintas, la verdad es que existe un considerable cuerpo de conocimientos que concuerdan con la existencia de tres a cinco dimensiones en culturas distintas y en fases evolutivas distintas ${ }^{3}$; que existe teoría capaz de explicar la mayor parte de los resultados alcanzados aunque, también es

2. Una notable excepción a todo ello lo representa la obra del desaparecido H. J. Eysenck que trató de aislar los componentes (y antecedentes) de personalided de los trastomos mentales. En este senttdo, el modelo de Eysenck no "tlene" aplicaciones a los trastornos de personelidad porque los contempla desde es inicio, aunque con una óptica heredada de los dos grandes grupos de síndromes en los que se dividia la psicopatologia: neurosis y psicosis.

3. La cuestión acerca del "número de dimensiones" tiene une doble consideración: por una parte, el problema de la omnitud (conocer todas las dimensiones básicas); por otra, el problema de la utilidad. No tiene sentido criticer la psicologla de la personalidad porque no se conocen todas las dimenslones. Lo que importe es que las conocidas sean relevantes. O dicho de otro modo, serla como rechazar la exdstencia y relevancia de los neurotransmisores porque no se conocen todos, o de los elementos quimicos porque hay huecos en las tablas clasificatorias. Una cosa es "conocer todo" (que sería una pretensión metafisica) y otra muy distinta, que lo que se conoce sea relevante (lo que resulta una cuestión cientfica). 
verdad que la psicología de la personalidad representa una especialidad compleja y un tanto difícil, y que exige una especial preparación metodológica y teórica. Además, existe una pluralidad de modelos científicos, lo que permite la contrastación de derivaciones sustantivas de ellos y que los instrumentos que produce cumplen los criterios psicométricos de bondad al uso.

Por otro lado, es posible pensar, como se apuntó más arriba, que se trate de un problema de "criterio", o lo que es lo mismo, que los marcadores diagnósticos utilizados para la evaluación de los trastomos de personalidad no sean claros y diferenciadores: (a) el diagnóstico categorial utilizado ha dado lugar a coeficientes de concordancia entre jueces muy mejorables; (b) la estabilidad del diagnóstico se ha modificado en períodos distintos; (c) la coexistencia de más de un trastorno de personalidad en cada uno de los pacientes diagnosticados de manera que existe una evidente y clara "comorbilidad" tanto en el sistema de la CIE como en los sucesivos DSM ( $y$ por los datos que ha recogido el autor senior, más en el segundo sistema y en la última versión que en el de la Organización Mundial de la Salud); (d) finalmente, dada la lógica diagnóstica utilizada (basta con la presencia de tres o cuatro criterios para un diagnóstico) es posible que con una misma denominación se encuentren persoras con una sintomatología distinta. De hecho, aplicando la lógica de variaciones con repetición se han llegado a proponer más de 100.000 formas distintas de recibir un diagnóstico de trastorno antisocial de la personalidad y más de 1000 de trastorno límite. $Y$ ello, obviamente, dificulta la obtención de un diagnóstico de "homogeneidad" de pacientes, dificulta la formación de grupos homogéneos para llevar a cabo ensayos clínicos controlados y, en definitiva, llevar a cabo estudios coherentes y sistemáticos.

Frente a estas críticas hay que decir que es verdad la detección de graves carencias en evaluación, etiología, entidad clínica, tratamiento y pronóstico. Pero que, a pesar de ello, se está utilizando el diagnóstico, que se ha avanzado en el conocimiento de estas entidades nosológicas y que solamente a base de estudio y acumulación de resultados y de intentos teóricos es posible un avance significativo en el conocimiento. Como reza un adagio oriental, "Lo 
mejor es enemigo de lo bueno" y se debe funcionar con lo que tenemos con el fin de poder mejorar la situación.

Lo que parece ser aceptado por muchos investigadores es que existe un problema de relación entre personalidad y trastomos de personalidad y que los intentos realizados hasta el momento no son satisfactorios (teoría de facetas, búsqueda de dimensiones alternativas, etc.). Dos hipótesis básicas se manejan en este trabajo: por una parte, la idea de que una parte sustantiva de trastornos de personalidad recogidos en la CIE-10 se refieren a aspectos centrales de lo que antes se denominaban personalidades neuróticas $y$, en especial, de aquellas que eran muy resistentes a los tratamientos, otras que estaban entre lo que se denominaba neurosis y psicosis y resultaban pacientes especialmente difíciles de manejar (como es el caso de los llamados trastomos límite de la personalidad) y otros, finalmente, que presentaban cuadros cercanos a las psicosis aunque no llegaban a presentarios de forma nítida de ellas. En este sentido, una de las tradiciones de investigación y que se encuentra presente en muchos de los casos es la ansiedad, denominada a veces inestabilidad emocional y otras, en contextos clínicos cuando se trataba de casos graves, de angustla. Con variaciones considerables en conceptuación, la ansiedad (en versión de personalidad, el neurotlcismo) representa una de las dimensiones de personalidad que no son discutidas por ninguno de los autores. En el modelo de parámetros (Pelechano, 1997, 2000; Pelechano y Servando, 2004) se diferencia entre ansiedad temperamental, con claro asentamiento biológico, estable y consistente, de la ansiedad de nivel de consolidación intermedio (ansiedad contextual) y, ésta, a su vez, de la ansiedad situacional dependiente de situaciones concretas.

En este trabajo se trata de ansiedad temperamental básica y en este sentido se predicen relaciones positivas entre ansiedad y trastornos de personalidad. Estas relaciones deberian ser muy altas en el caso del trastomo ansioso, y límite en la medida en que existe tradición teórica que sitúa a estos dos trastomos en una forma de trastornos de ansiedad graves; relaciones un poco menos intensas cabría esperar en los trastornos impulsivo (fuerte componente de ansiedad generalizada), dependiente (con fuerte componente de ansiedad social al menos), histrionico (ansiedad social y una fuerte 
tradición teórica que o relacionaría como una de las múltiples formas de la antigua histeria) $y$, en todo caso, por el componente de ansiedad social intensa presente, el trastorno paranoide. Finalmente cabrían esperar relaciones tenues con el trastorno disocial (no sería neuroticismo sino la dimensión de psicoticismo la que se encontraría presente en este trastorno) y prácticamente ausentes en el trastomo esquizoide (sería la dimensión de introversión-extraversión la que se encontraría muy relacionada en este caso y no la de neuroticismo). Estas relaciones pueden ser moduladas por ciertos criterios de identificación tales como nivel profesional, edad y género, aunque de entrada cabría esperar coeficiente de menor cuantía en los análisis moduladores correspondientes a estos análisis zonales (por restricción de varianza) a menos que se encontrasen "bolsas" de submuestras con características específicas que representen grupos con una gran vulnerabilidad a sufnir de algunos trastomos de personalidad en los que el neuroticismo desempeñe un papel especial.

Hasta aquí tipos de previsiones generales que pueden ser consideradas de corte tradicional en cuanto a análisis. En la medida en que la consistencia interna de los criterios definicionales de los trastornos sea baja, es posible pensar que las correlaciones directas empíricas se encuentren sesgadas o incluso disminuidas debido a esta inconsistencia y por ello, la conveniencia de llevar a cabo correcciones por atenuación, con el fin de acercarse a las "relaciones reales" entre dimensiones de personalidad y criterios de identificación de trastomos de personalidad.

De manera más especifica, los objetivos que se persiguen en este trabajo son los siguientes: (a) comprobar si las puntuaciones altas en la escala de neuroticismo de H. J. Eysenck predisponen a padecer alguno de los trastornos de personalidad propuestos por la CIE; (b) aislar los criterios definitorios de los trastornos que se relacionen más con neuroticismo y, a partir de aquí, descubrir si algunos de los elementos definitorios de los trastomos son más propios de neuroticismo que de cada uno de los trastornos evaluados (redundancias conceptuales y empíricas) y (c) estudiar el papel diferencial que desempeñan la edad, el género y el nivel profesional en las relaciones que existen entre neuroticismo y los distintos trastomos de personalidad. 


\section{MÉTODO}

\section{(a). Muestra}

Ha participado una muestra incidental no clínica formada por 793 personas españolas (623 residentes en la isla de Tenerife y 170 en Valencia). El $62,9 \%$ de ellas mujeres. El rango de edad ha sido de 18 a 74 años (media de 35,4 años y desviación típica de 11,22); todas ellas cumplimentaron una prueba sobre trastomos de personalidad (véase más abajo). Aproximadamente la tercera parte de la muestra eran solteros $(35,1 \%)$, el $38,7 \%$ casados o vivian con su pareja; 23 de la muestra estaban separados o divorciados y el 0,6\% eran viudos. Por lo que se refiere a nivel profesional/ocupacional, el 1,4\% estaban en situación de paro laboral, el 62,9\% desempeñaba un trabajo manual (cualificado o no, poco más de la mitad de esta categorla con cualificación profesional), el $11,1 \%$ eran estudiantes universitarios y el $0,4 \%$ estaban jubilados.

Dentro de este grupo se distingue una submuestra formada por 459 personas que cumplimentaron además, la escala de neuroticismo (véase más abajo). Esta submuestra se encuentra formada por 294 mujeres $(64,1 \%)$, con rango de edad entre $18^{-}$y 64 años media similar (moda entre 25 y 44 años, el $58,8 \%$ ), el $29,2 \%$ solteros y el $42 \%$ casados o viviendo en pareja, el $3 \%$ separados divorciados y un viudo. El $68,4 \%$ eran trabajadores manuales cualificados o no, el $2,8 \%$ con profesiones liberales y el $9,2 \%$ estudiantes.

No habia noticia, en el momento del pase de pruebas, de que los participantes sufriesen de algún trastomo mental. Se trata, en este caso, de un estudio de análogos en el que se asume que la relación entre personalidad y trastornos de personalidad se mantiene constante a todo lo largo de las puntuaciones en los dos polos de la relación. Existe cierta evidencia que avala este supuesto. Deary y Meter (1998), Livesley et al. (1998) encontraron que la estructura de los trastornos de personalidad y de las dimensiones de personalidad son similares en muestras clínicas y no clínicas, así como que las diferencias entre ambas son más cuantitativas que cualitativas, que las tasa de comorbilidad obtenidas con el uso de cuestionarios son similares entre trastornos de personalidad (Watson y 
Sinha, 1998). Por otra parte, Lenzenweger, Loranger, et al. (1997) informan de coeficientes de consistencia interna por encima de 0,80 para grupos de trastornos de personalidad y que la mayoria de loso casos detectados como posibles trastornos de personalidad con el cuestionario IPDE-S - que hemos utilizado nosotros- fueron posteriormente diagnosticados como que sufrían de trastomos de personalidad posteriormente en la entrevista clínica.

\section{(b). Instrumentos}

Como marcador de neuroticismo se utilizó la escala de neuroticismo propuesta por H. J. Eysenck (EPI, 1964), traducida, adaptada y depurada por el autor senior desde 1970. Está formada por 20 elementos de respuesta obligada (verdadero falso), con formulación auto-referente y que ha mostrado su utilidad tanto en poblaciones clínicas como no clínicas. Ejemplos de ítems de los que componen la escala son los siguientes. "A menudo me siento de mal humor, desconcertado", "A veces tengo ideas que me vuelven una y otra vez a la cabeza". Evalúa sintomatología de ansiedad e inestabilidad emocional (gobernada en gran parte por el sistema neurovegetativo) y la teoría que se encuentra a la base defiende que las personas que puntúan alto en esta escala son vulnerables a trastomos de ansiedad. La evidencia experimental y correlacional disponible sugiere que no diferencia entre vulnerabilidad a trastornos de ansiedad y no vulnerabilidad pero que no diferencia en la vulnerabilidad a los distintos trastornos de ansiedad.

Se utilizó el cuestionanio IPDE ICD-10 de Loranger, Janca y Sartorius (1997), instrumento elaborado a petición de la Organización Mundial de la Salud, desarrollado en una ambiciosa investigación dirigida por Loranger y encaminada a ofrecer un instrumento criterial que sirviera como primer escrutinio de los trastornos específicos de la personalidad que se Identifican en la CIE-10 (ICD-10). Está basado en un instrumento anterior del propio Loranger (1988). Se trata de un conjunto de escalas criteriales formadas por un total de 59 elementos de respuesta obligada (verdaderoffalso) y se pide a los participantes que cumplimenten el cuestlonario teniendo en cuenta su manera de ver las cosas en los últimos cinco años. El 
cuestionario cubre los nueve trastornos especíicos de personalidad que se encuentran en la ClE-10 (paranoide, esquizoide, disocial, impulsivo, IÍmite, histriónico, anancástico, ansioso y dependiente). El número de items que se emiten para cada trastorno no es el mismo (entre cinco para el impulsivo, límite, ansioso y dependiente y nueve para el esquizoide).

Las puntuaciones en este cuestionario son insuficientes para la emisión o no de un diagnóstico de trastomo de personalidad. Para ello se exige la realización de una entrevista clínica posterior, deseablemente con el posible paciente y, además, otra con un familiar o persona significativa para el posible paciente que lo conozca bien.

\section{(c). Procedimlento}

Todos los participantes han sido colaboradores voluntarios. Por lo que se refiere a los estudiantes, la colaboración se premiaba con una pequeña bonificación ( 0,5 puntos en la nota final de prácticas de la materia Psicología de la Personalidad en la Universidad de La Laguna). Además, se pedla a los estudiantes que pidieran a familiares, amigos o pareja, la cumplimentación de las pruebas. Una vez cumplimentadas, se cerraban en un sobre y se remitian por correo al Departamento de Personalidad, Evaluación y Tratamientos Psicológicos de la Universidad de La Laguna. Los datos recogidos se codificaban con el fin de preservar la intimidad y se podía tener información comentada de los resultados individuales si así lo pedían, siempre que lo pidieran de forma individual y previa identificación, en entrevista personal con el autor senior de este trabajo. Los datos fueron calculados con el paquete SPSS-11 para PC.

\section{RESULTADOS}

(1).- Relaciones entre neurotlclsmo y escalas de trastornos de personalldad

El primer resultado se refiere al análisis directo de las relaciones entre la escala de neuroticismo y las escalas de trastornos de 
personalidad. Se ha dicho más arriba que la relación entre ambas series de varlables depende de la consistencia-fiabilidad de las escalas utilizadas. En la medida en que estos coeficientes son bajos, resulta conveniente utilizar la corrección por atenuación para acercarse a la "correlación real"entre neuroticismo y las distintas escalas de trastomos de personalidad. En la tabla 1 se encuentra recogidos los coeficientes de consistencia interna de neuroticismo y de las distintas escalas de trastomos, asl como la correlación entre neuroticismo y trastornos, con coeficientes corregidos por atenuación

Tabla 1.- Coeficlentes de consistencla Interns (a) de las escalas criteriales de trastornos de personalldad y de neuroticlsmo. En la segunda flla los coeficlentes de correlación de Pearson entre escalas de trastornos de personalldad y neurotlclsmo. En la tercera flla los coeflclentes de correlaclón corregldos por atenuaclón.

En la tabla F60.0= paranolde; F60.1=esqulzolde; F60.2= dlsocial; F60.30= Impulsivo; F60.31 = límlte; F60.4= hlstrlónlco; F60.5= anancástlco; F60.6= ansloso; $F 60.7=$ dependlente.

En la tabla se han omitldo los ceros y se han sustltuldo por un punto en los coeficlentes. La expllcación en el texto.

\begin{tabular}{|l|l|l|l|l|l|l|l|l|l|}
\hline Criterio & $F 60.0$ & $F 60.1$ & $F 60.2$ & $F 60.30$ & $F 60.31$ & $F 60.4$ & $F 60.5$ & $F 60.6$ & $F 60.7$ \\
Dimension & $\alpha=.39$ & $\alpha=.44$ & $\alpha=.37$ & $A=.45$ & $\alpha=.32$ & $a=.39$ & $\alpha=.54$ & $\alpha=.46$ & $\alpha=.49$ \\
\hline Neuroticismo & .38 & -.18 & .13 & .36 & .38 & .27 & .29 & .56 & .39 \\
\hline$\alpha=.82$ & .66 & -.36 & .29 & .59 & .94 & .55 & .48 & .98 & .65 \\
\hline
\end{tabular}

La consistencia interna de neuroticismo es, alta $(0,82)$. Frente a ello, los coeficientes alfa correspondientes a cada uno de los trastornos tienen un valor que es la mitad o poco menos del correspondiente a neuroticismo, lo que indicaría una considerable variabilidad de respuesta dentro de cada escala (heterogeneidad de respuesta). 
Los resultados confirman en gran medida las previsiones que se han formulado más arriba:

(a).En primer lugar, los coeficientes correspondientes al trastorno ansioso y al límite son muy altos (0,98 y 0,94 respectivamente). Ello sugeriria que el trastomo ansioso prácticamente es lo mismo que la escala de neuroticismo y algo menos (pero muy poco menos) por lo que se refiere al trastorno límite de personalidad. Repárese que en la escala de neuroticismo se $\mathrm{H}$. J. Eysenck que hemos utilizado se encuentran presentes tanto ítems correspondientes a ansiedad como a depresión, y esta "impureza" sería, en parte, la responsable de los resultados alcanzados. De rechazo, estos resultados sugeririan que cuando una persona presenta puntuaciones altas en la escala de neuroticismo, habria que investigar la posible cornorbilidad de estos dos trastornos de personalidad.

(b).Un segundo grupo de trastornos presenta coeficientes entre 0,55 y 0,66 : el trastorno paranoide $(0,66)$ y posiblemente correspondiente a la ansiedad social, el trastomo dependiente $(0,65)$ asimismo por ansiedad social, aunque en este caso seria a quedarse solo/a, el trastorno impulsivo $(0,59)$ en el que parte de la falta de control impulsivo sería debida a ansiedad-premura por acabar las cosas y vencer la resistencia de los demás $y$, finalmente, el trastorno histriónico $(0,55)$ heredero de componentes de la desaparecida histeria.

(c).A corta distancia se encuentra la relación de 0,48 entre neuroticismo y trastorno anancástico debido posiblemente a la parte de este trastorno que se identifica con el detallismo y adhesión rígida a normas, rituales y formalismos (lo que lo acerca a una sensibilidad autistoide), si bien el porcentaje de varianza compartida sugiere que se trata de un aspecto funcional no identificable con neuroticismo.

(d).Finalmente el coeficiente correspondiente al trastomo disocial es bajo $(0,29)$, lo que significaría que este trastorno poco tiene que ver con la ansiedad. El coeficiente correspondiente al trastorno esquizoide es incluso negativo y de intensidad similar al encontrado con el trastorno disocial $(-0,36)$, lo que sugiere que se trata fundamentalmente de otro tipo de alteración y en el que la ansiedad desempeña un papel muy escaso y apenas significativo (menos del $13 \%$ de varianza compartida en situación ideal). 


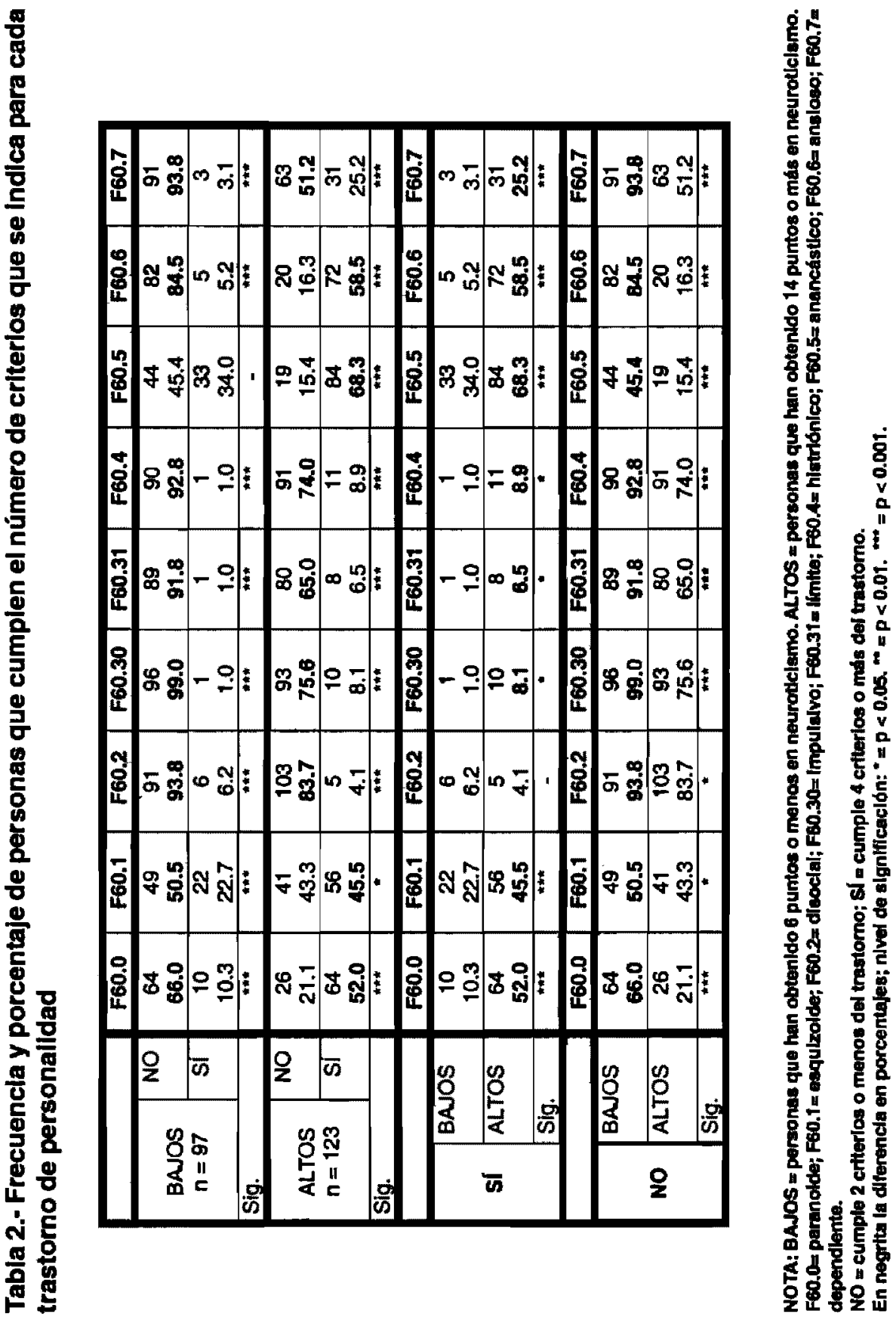


Con el fin de seguir en la línea de discurso de apresar la covariación entre neuroticismo y trastornos, en la tabla 2 se presenta un conjunto de resultados desde dos puntos de vista. En la mitad superior (columnas con bajos y altos) se han separado a los participantes en dos grupos apelando a las puntuaciones que han alcanzado en la escala de neuroticismo (25\% superior e inferior, que corresponde, el primer grupo a puntuaciones hasta 6 puntos de un total posible de 20 y el segundo a puntuaciones iguales o superiores a 14 puntos).

Por otra parte se ha dicotomizado la parte correspondiente a las puntuaciones en las escalas de trastornos de la siguiente manera: "NO" corresponde a personas que poseen dos menos de los criterios identiflcativos de cada trastomo (a menos que hayan mentido este grupo resulta muy poco probable que recibe un diagnóstico "positivo" del trastorno de personalidad correspondiente); "SI" corresponde a los participantes que han alcanzado una puntuación de 4 o más dentro de cada escala de trastorno y, usualmente, tienen un riesgo alto de recibir el diagnóstico del trastorno de personalidad en cuestión después de la entrevista de evaluación.

Los resultados de la parte superior de la tabla (filas bajo los ep(grafes "bajos" y "altos") se refieren a número absoluto de personas dentro de la submuestra en la primera fila y, en la segunda, el porcentaje correspondiente. La fila de "bajos/si" recogeria los "falsos positivos" de la submuestra en el análisis (personas que puntúan bajo en neuroticismo mientras que puntúan alto en cada trastorno) y la fila de "altos/no"serían los "falsos negativos" (personas que puntúan alto en neuroticismo y bajos en cada uno de los trastomos). En la mitad inferior de la tabla se encuentran los mismos resultados si bien ordenados de otra manera: "Si" se refiere a personas que han puntuado alto en cada trastorno ( $>4)$ y "No" a personas que han puntuado bajo en cada trastorno $(<2)$. Bajos y altos se refiere, respectivamente, a puntuaciones del primero y del cuarto cuartil en neuroticismo. En las dos mitades de la tabla se han calculado las diferencias en porcentajes de las celdillas correspondientes a las comparaciones y señalado el nivel de significación estadístico de las diferencias. Se han puesto las dos partes de la tabla con el fin de ofrecer un panorama más claro de los resultados alcanzados sobre este punto: 
(a). En primer lugar, una llamada de atención acerca del trastorno disocial: se trata de una muestra de personas que viven en comunidad, normalmente socializadas, por lo que no caben esperar muchos casos de participantes con una puntuación en la escala de 40 más puntos (de hecho, 11), por lo que las comparaciones en este trastorno deben tomarse con especial cautela.

(b). En segundo lugar la tendencia es muy clara y coherente en el caso de puntuaciones bajas en neuroticismo. Excepto en el caso del trastorno anancástico (F60.5), las diferencias en porcentajes son todas estadísticamente significativas y a favor de la hipótesis de que unas puntuaciones bajas en neuroticismo servirian para "galvanizar" o proteger de los trastornos de personalidad (grupo de personas con baja puntuación en neuroticismo). Los que presentan alto riesgo de trastorno de personalidad mientras puntúan bajo en neuroticismo (falsos negativos) son más probable que ocurran en el trastorno anacástico $(34 \%)$ y esquizoide $(22,7 \%)$ y menos en el impulsivo, límite e histriónico (1\%), y después, en el ansioso $(5,2 \%$ y en el dependiente $(3,1 \%)$.

(c). En tercer lugar, los "errores" son porcentualmente mayores en el caso del grupo con puntuaciones altas en neuroticismo. En el trastorno disocial $(83,7 \%)$ es tan grande que prácticamente parecería funcionar como el reverso de la predicción directa (a menor puntuación en la escala del trastorno, mayor porcentaje de personas con puntuaciones altas en neuroticismo), lo que sugiere que la dimensión de neuroticismo representaría, en el mejor de los casos, una "protección" frente a este trastorno y, de hecho, sería irrelevante o, en el mejor de los casos, un indicador de que se debe buscar en otra parte.

El porcentaje de falsos negativos (personas con grave riesgo de sufrir un trastorno y que no son identificados solamente con la dimensión de neuroticismo), aparte el trastorno disocial es muy alto en el trastorno impulsivo (75,6\%), límite $(65 \%)$ e histriórico $(74 \%)$ $y$ es superior al $40 \%$ en el esquizoide y el dependiente. Entre el 45 $y$ el $68 \%$ de aciertos diagnósticos se encuentran el esquizoide, paranoide, anancástico y ansioso.

Estos resultados representarían una fuerte llamada de atención respecto a la utilización de solamente una dimensión y metodología 
(la correlacional) para la investigación y mejora de los niveles predictivos de los trastornos de personalidad a partir de las dimensiones de personalidad. Por otro lado, estos resultados representan un recordatorio de que los trastornos de personalidad, tal y como se encuentran definidos oficialmente con las escalas criteriales, representan más de una dimensión de personalidad, al menos en su mayor parte, a la hora de la evaluación diagnóstica. $Y$ finalmente, la existencia de posibles asimetrlas en la precisión evaluativa (falsos negativos y falsos positivos) de las dimensiones de personalidad $y$, de rechazo, en la supuesta linealidad de las relaciones entre dimensiones y escalas de trastornos.

(2). Un rastreo acerca de ftems de escalas de trastornos cercanos a la "dimensión" de neuroticismo

La variabilidad en las relaciones entre escalas de trastornos y neuroticismo implica que no todos los ítems de las escalas se encuentran igualmente comprometidos con la dimensión de personalidad utilizada. Y ello querría decir, asimismo, que puedan existir unos ítems de las escalas que sean los "responsables" de esta relación. Con el fin de apresar los ftems que mejor recogen la dimensión de neuroticismo presente en las escalas de trastornos en el cuestionario de Loranger se operó de la manera siguiente. En primer lugar se llevó a cabo un análisis factorial (extracción de componentes principales, petición de un solo factor) de los ítems de cada escala de trastornos y de neuroticismo, lo que hacen un total de nueve análisis. En segundo lugar, se seleccionaron aquellos ítems de las escalas criteriales que saturaban igual o mayor a 0,30 en el factor aislado en cada análisis factorial. Se encontraron un total de 20 items.

Finalmente, se llevó a cabo un análisis factorial conjunto de estos ítems seleccionados. Se pidió una extracción monofactorial (componentes principales) y se seleccionaron los que ofrecieron un peso factorial igual o superior a 0,30. Los resultados se recogen erı la tabla número 3. 


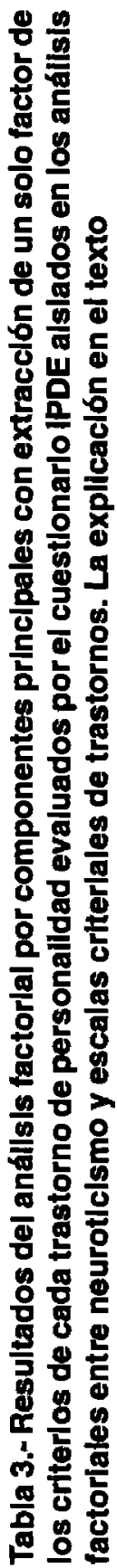

\begin{tabular}{|c|c|c|c|c|c|c|c|c|c|c|c|}
\hline$x$ & $\mp$ & ભ & 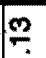 & ক্. & চ্ & $\stackrel{n}{?}$ & $\div$ & $\stackrel{\Phi}{?}$ & $\mp$ & $\stackrel{20}{?}$ & |ֶ \\
\hline 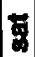 & మ్లి & to? & 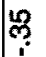 & 岩? & ? & ?ִ & 岁 & Z & ชె. & 爯 & $\bar{\varphi}$ \\
\hline 8 & $\tilde{q}$ & P. & 운 & 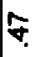 & ำ & 果 & ధి & \% & ४ & 운 & q \\
\hline$x$ & శֶ & $\mathbb{v}$ & g & พั & 19 & œִ & 9 & 오 & $\mathbb{g}$ & 罗 & 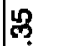 \\
\hline & 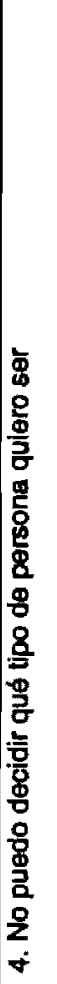 & 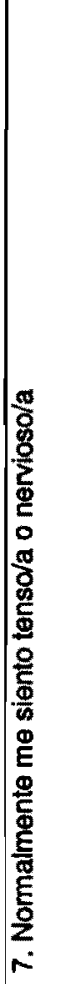 & 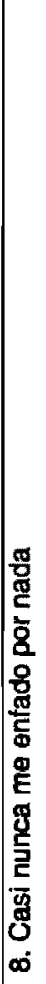 & 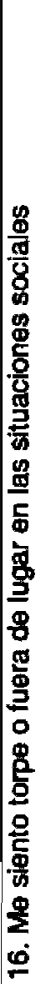 & 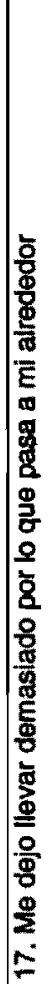 & 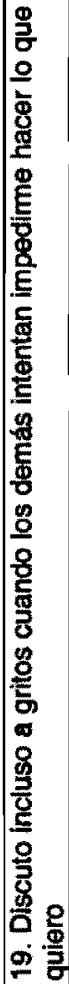 & 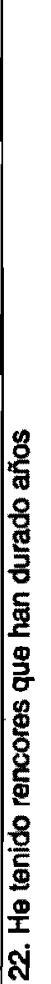 & 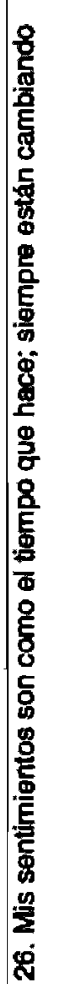 & 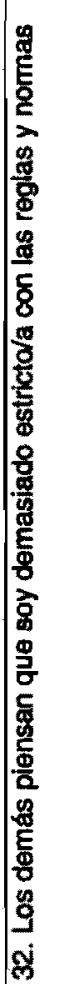 & 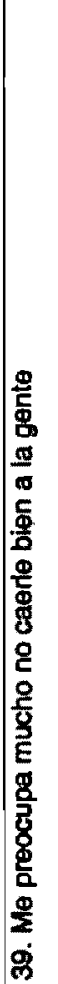 & 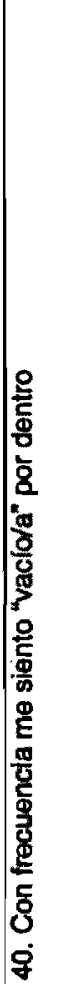 \\
\hline & & & & & & 8 요 & & 通 & & 8 & 8 贯 \\
\hline
\end{tabular}



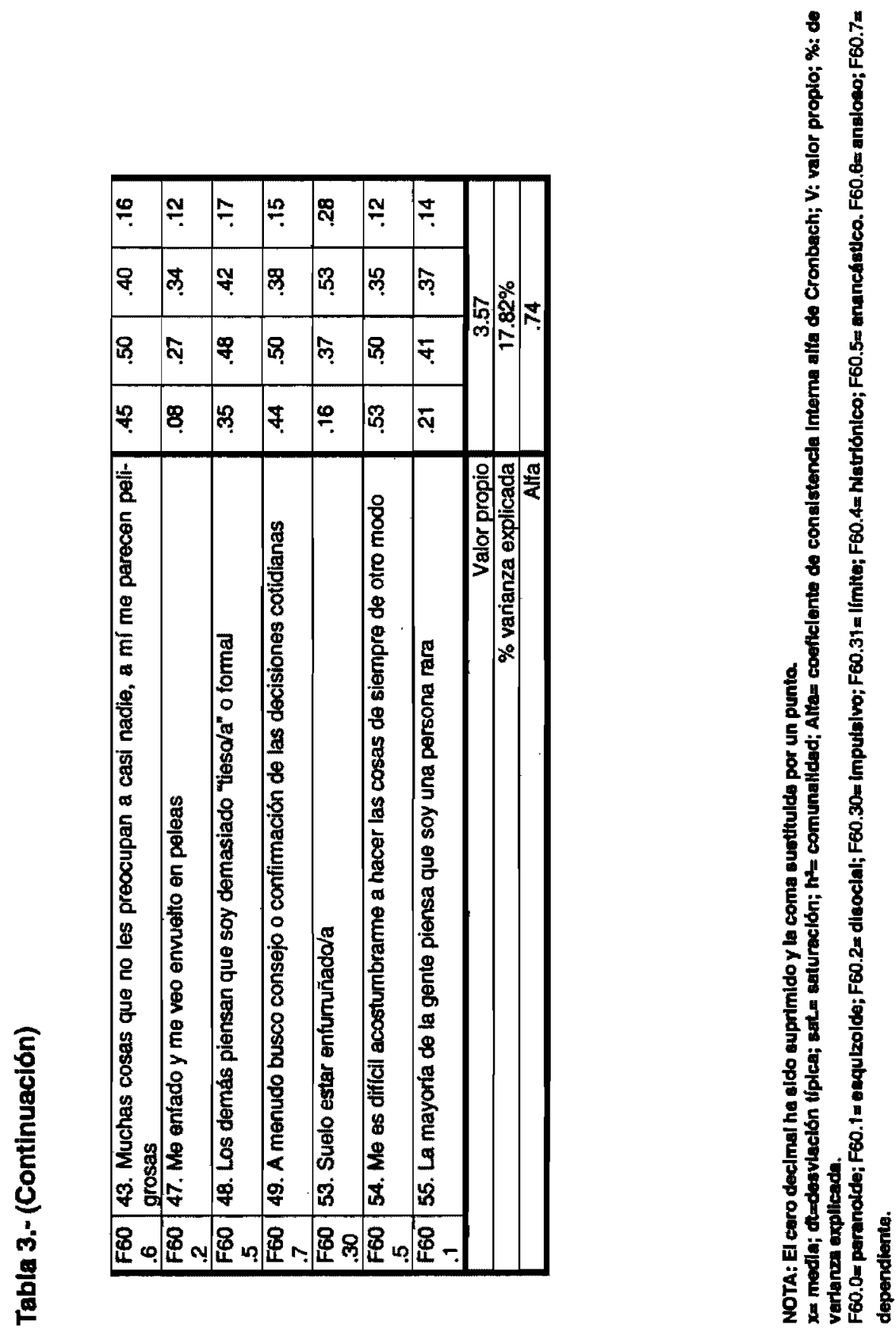
En la primera columna la identificación de los ítems respecto a la escala de trastorno correspondiente. El factor aislado presenta una consistencia interna (alfa) de 0,74 y explica cerca del $18 \%$ de la varianza total. Como cabría esperar, el trastorno que "aporta" mayor número de ítems (4) el trastorno ansioso, seguido del anancástico (3), con dos ítems impulsivo, IImite, esquizoide (un ftem con peso negativo) e histriónico; y con un ítem, el paranoide, disocial y dependiente.

El análisis del contenido de los ítems sugiere que se trata de tres núcleos significativos. El primero y de mayor peso se refiere a cuestiones relacionadas con irritación, enfado y adhesión a normas y rutinas de forma rígida; el segundo y unido al anterior, se refiere a ansiedad social o elementos de inestabilidad emocional y el tercer núcleo a sentimientos de unicidad, y ser distinto al grupo, con algún componente depresivo (como sentimiento de vacío personal). Todos ellos pueden ser interpretados como inseguridad social y personal $y$, en este sentido "hace sentido" que forme parte de la inestabilidad emocional, que a su vez se relaciona estrechamente con neuroticismo. Es posible interpretar este factor como la parte de ansiedad presente en los distintos trastornos de personalidad $y$, a la vista de los resultados, neuroticismo no se encuentra representado con el mismo peso en los distintos trastornos de personalidad propuestos por la CIE-10.

(3). Moduladores diferenclales de la relación entre neurotleismo y trastornos de personalidad

La muestra que ha participado en este estudio ha sido amplia y por ello es posible que las relaciones encontradas hasta ahora escondan unas modulaciones que podrían descubrirse con análisis zonales de la muestra. Nos ocupamos en la presentación de las relaciones entre neuroticismo y las distintas escalas criteriales de trastomos de personalidad. En la medida en que no todos los grupos formados poseen el mismo número de participantes, el procedimiento seguido ha sido el siguiente: comparación de coeficientes de correlación entre subgrupos distintos (por edad, género, nivel pro- 
fesional) y para ello, transformación de los coeficientes de correlación a puntuaciones " $\mathrm{z}$ ", para pasar, después, a las comparaciones entre coeficientes de correlación.

\section{3.a. La evolución de la relaclón entre neuroticlsmo y trastornos de personalldad}

No existen datos que permitan establecer patrones empíricos evolutivos acerca del peso diferencial que tiene el factor de neuroticismo-ansiedad básica en los distintos trastornos de personalidad. Si bien los datos que hemos recogido no permiten un estudio longitudinal, los análisis trasversales podrian generar hipótesis e iluminar to que cabria esperar en poblaciones clínicas.

De entrada hay que subrayar que se trata de unas primeras estimaciones y su significación va en la línea del poder de "predicción" y de comprensión de neuroticismo en los distintos trastornos de personalidad.

Una primera inspección de los resultados obtenidos permitiría agrupar los trastomos en tres grupos. Los dos primeros se recogen en la figura 1 y el tercero en la figura 2.

En el primer grupo se encuentra el trastorno ansioso: la tendencia observada es un incremento en el compromiso del neuroticismo a medida que se incrementa la edad (propiamente no es la edad, sino los sucesos que se dan en ese período temporal). Las diferencias entre los grupos de edad son estadísticamente significativas en todos los casos y la relación es más intensa a mayor edad (los valores de " $z$ " pasan de 1,53 a 2,64). Podría sugerirse que, a partir de los 40 años aproximadamente, el trastorno ansioso resulta en gran medida, un estado de ansiedad generalizado, con una base orgánica que sería responsable de casi la mitad de la varianza.

En un segundo grupo se encontrarian los trastomos impulsivo, histriónico y disocial. En este grupo o bien no existe un papel diferencial claro del neuroticismo a lo largo de la edad, como sucede en el trastorno impulsivo (ninguna diferencia estadísticamente significativa), o bien caben esperar relaciones en forma de U-invertida como en los trastornos histriónico y disocial. El perfil relacional del 

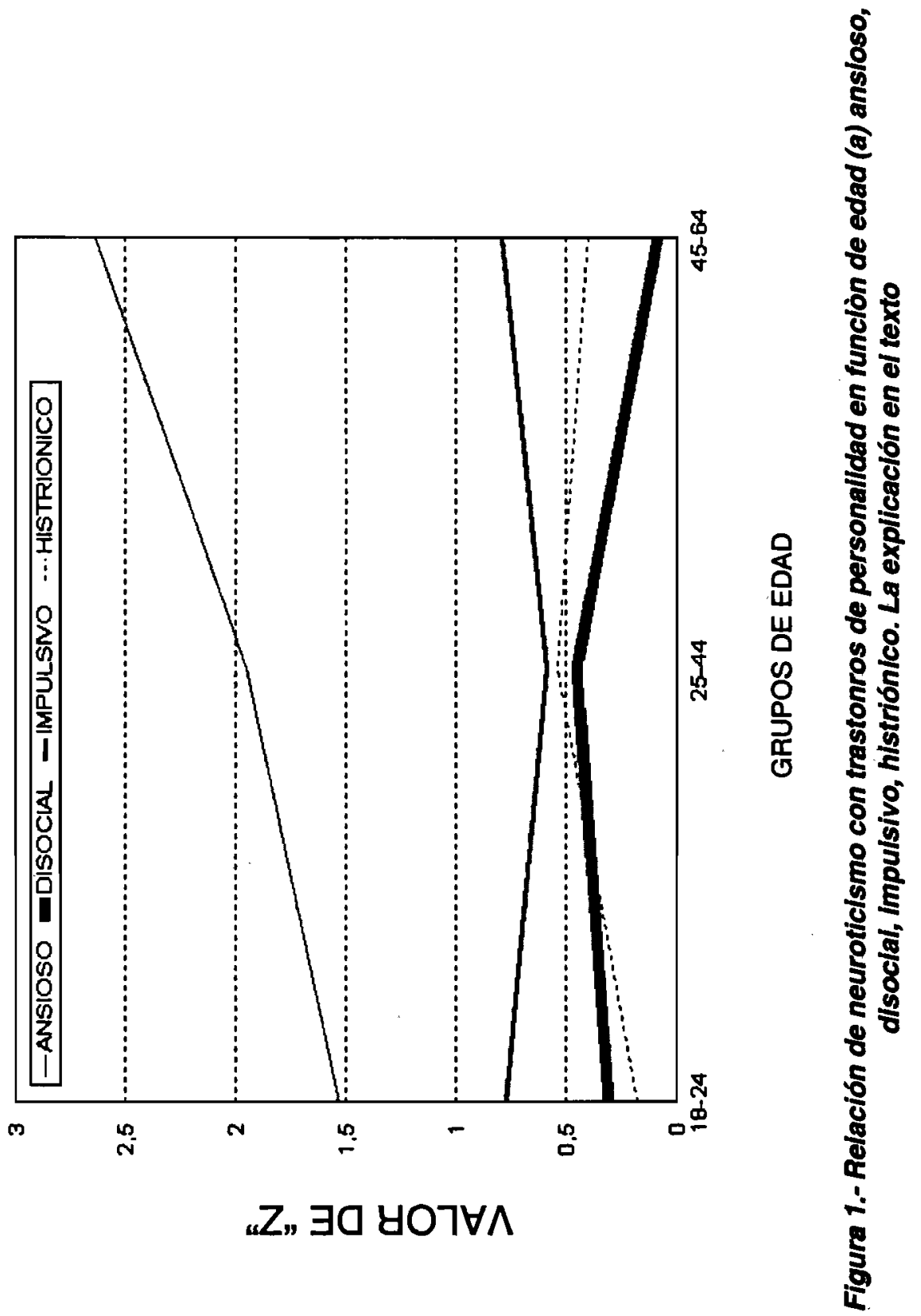


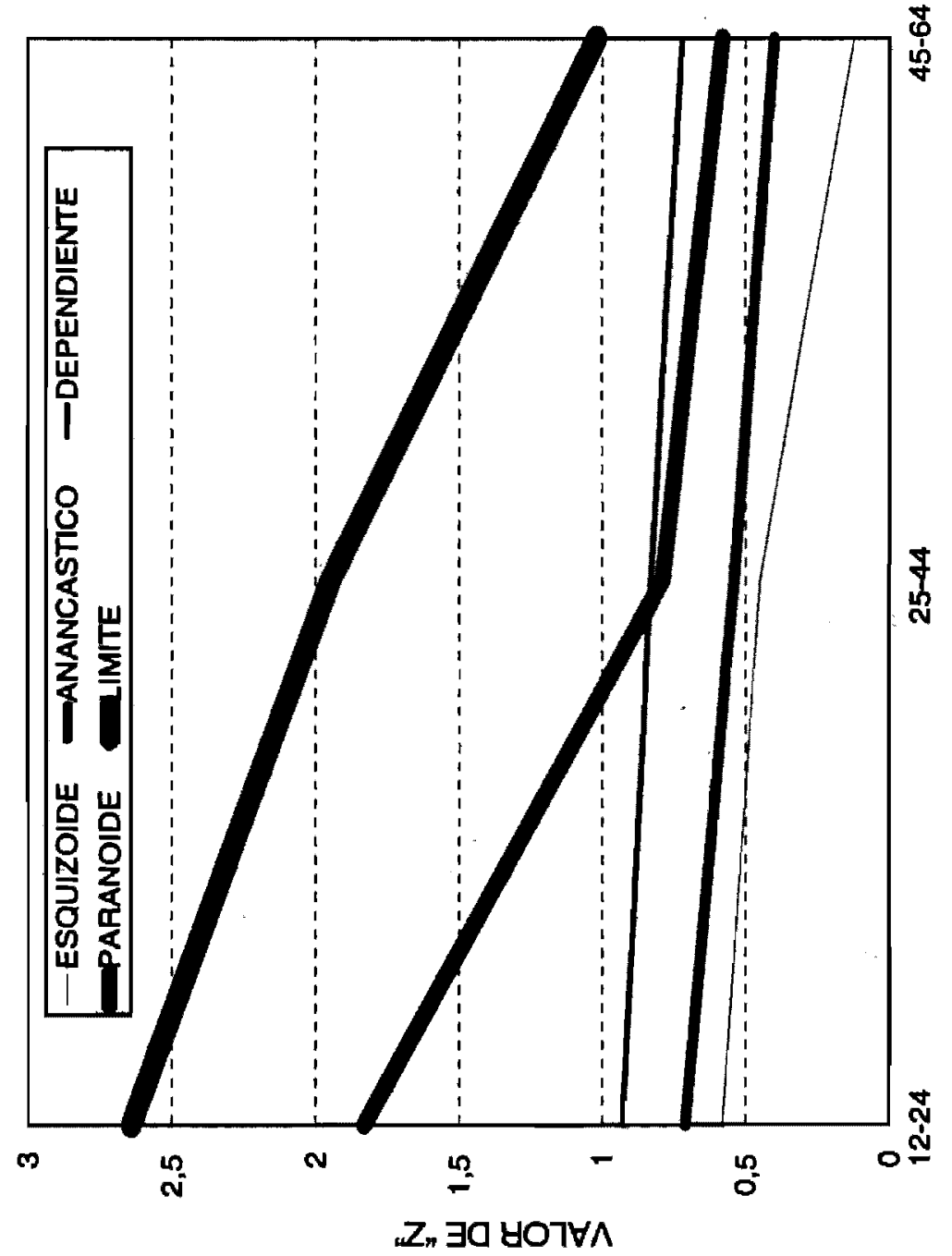

题

$\frac{0}{8}$

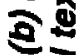

8

8

$\$ 5$

5

9

농

7 옹

4 Ш

山 홍

0 둥

옹

$\frac{1}{0}$

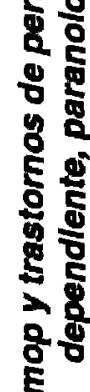

$\frac{5}{5}$

8 
neuroticismo con estos dos últimos trastomos es el mismo y, además, con parecidos niveles de significación estadistica en las diferencias: la máxima relación se obtiene en el período de edad de 25 a 44 años. La relación crece desde los 18-24 a los 25-44 ( $p<0,01$ en los dos trastomos) y disminuye desde los 25-44 a los 44-64 ( $<<0,05$ y 0,01 en uno y otro trastorno hasta casi desaparecer). Posiblemente una de las dificultades que existe entre el diagnóstico diferencial del trastorno histriónico y el disocial se deba a este patrón común de relación y que podrla ser debido a los papeles similares que juega la ansiedad en los dos casos. $O$, altemativamente, la existencia de un notable patrón de comorbilidad de ambos trastornos, con un mismo patrón evolutivo y cuya comorbilidad pudiese ser debida al neuroticismo común.

El tercer patrón evolutivo de la relación se encuentra recogido en la figura 2 en el que la relación entre neuroticismo y trastornos de personalidad va disminuyendo a medida que se avanza en edad. Puede diferenciarse dos grupos: en el primero (trastorno dependiente y anancástico) la relación va disminuyendo aunque no existen diferencias estadisticamente significativas de estos cambios (y asimismo, las relaciones tienden a no ser significativas). En el segundo grupo, formado por los trastornos limite $y$ paranoide la tendencia a disminuir la relación es notoria: en el trastorno límite el compromiso va disminuyendo y las diferencias son estadisticamente significativas $(p<0,001)$, en el trastorno paranoide la caída de la relación se da entre el período 18-24 años y el de 25-44 ( $p<0,001)$, y la caída se enlentece hasta el punto de que no es estadísticamente significativa. Se sugiere la hipótesis de que es este compromiso en común con neuroticismo el que explicaria la mayor parte de la comorbilidad entre trastorno paranoide y limite, y que esta comorbilidad tendería a desaparecer más allá de los 44 años.

Estos compromisos que se modifican a lo largo de un notable período del ciclo vital sugeririan que la personalidad cambia, y que puede modificarse, asimismo, en grandes período de tiempo, los compromisos y hasta elementos definitorios de lo que significan ciertos trastornos de personalidad. Las implicaciones para la evaluación y el tratamiento son indudables. 


\section{3.b. El papel diferenclal del género}

El análisis comparativo de la diferencia de coeficientes de correlación entre neuroticismo y escalas de trastomos en hombres (161) y mujeres (294) ha dado como principal resultado que en la mayoría de trastornos no se detectan relaciones diferenciales entre neuroticismo y trastornos de personalidad para hombres y mujeres. Las diferencias se han localizado en dos: límite y ansioso y se encuentran representadas en la figura 3.

La relación entre neuroticismo y trastorno límite es significativamente más alta $(p<0,001)$ en mujeres que en hombres. Se sabe, además, que este tipo de trastorno es más frecuente en mujeres que en hombres, que no existe una solución satisfactoria ni farmacológica ni psicológica y que la ansiedad y la depresión son componentes importantes de ese trastomo. Repárese que no se trata de que las mujeres presenten puntuaciones más altas en neuroticismo sino que la relación entre éste y el trastorno límite es significativamente mayor en las mujeres. Significaría que en los hombres la relación entre trastorno límite y neuroticismo es significativamente menor y por ello, posiblemente desempeñaría un papel menos relevante en la estructura y en la dinámica del trastorno.

En el trastomo ansioso, el fenómeno observado es justo el opuesto: mayor relación $(p<0,001)$ entre neuroticismo y trastorno ansioso en hombres que en mujeres. $O$ dicho con otras palabras: en los hombres el peso de la ansiedad es mayor que en las mujeres en el trastorno ansioso y, como sugerencia, la disminución de la ansiedad podría redundar en la disminución del trastorno o una mayor susceptibilidad a la mejoría, pero solamente en el caso de los hombres.

\section{3.c. El papel del estado clvil en la relación entre neuroticlsmo y trastornos de personalldad}

Se ha seguido el procedimiento descrito en el estudio de las diferencias entre solteros y casados. Los resultados significativos se encuentran representados en la figura 4. 


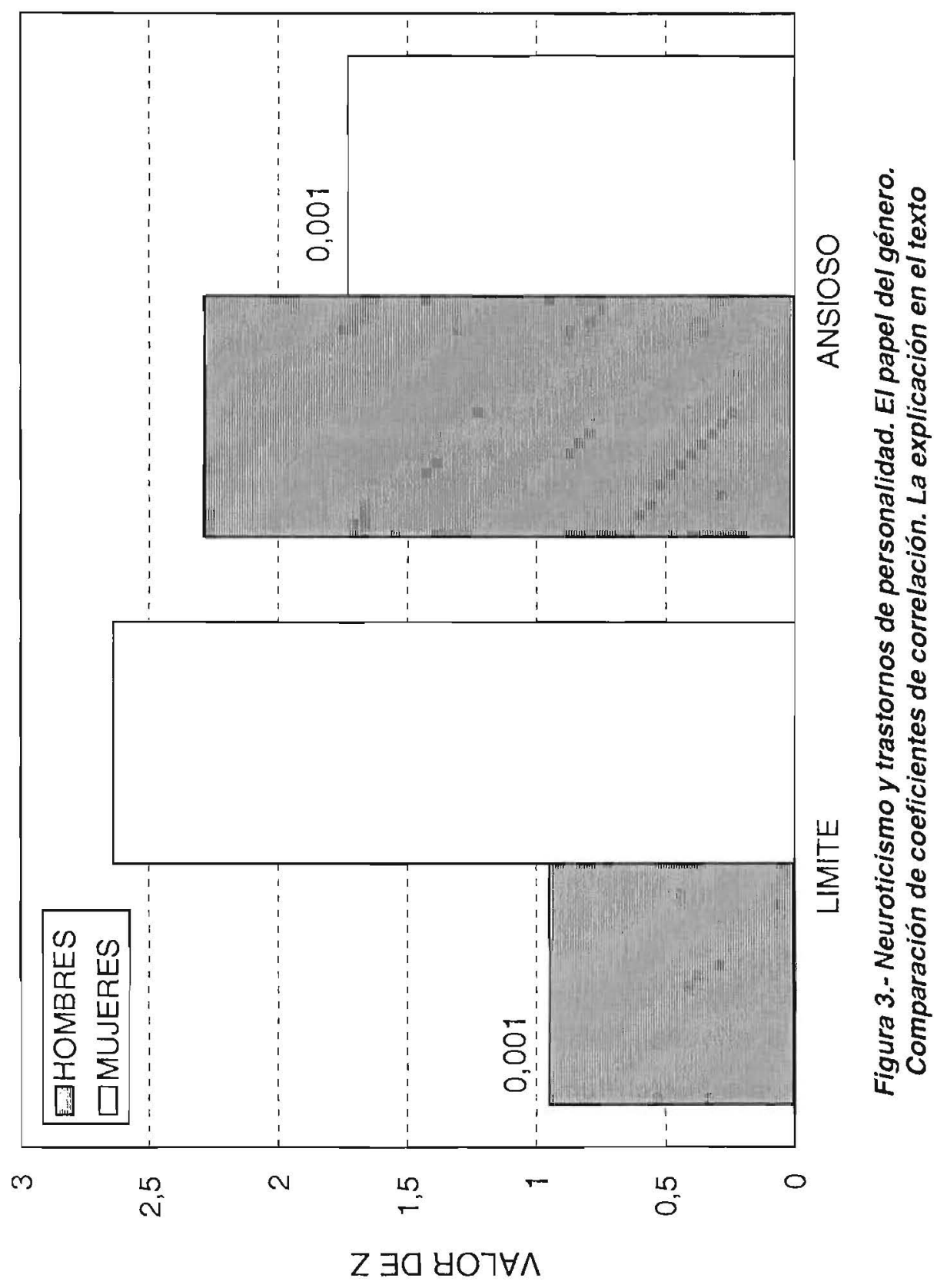




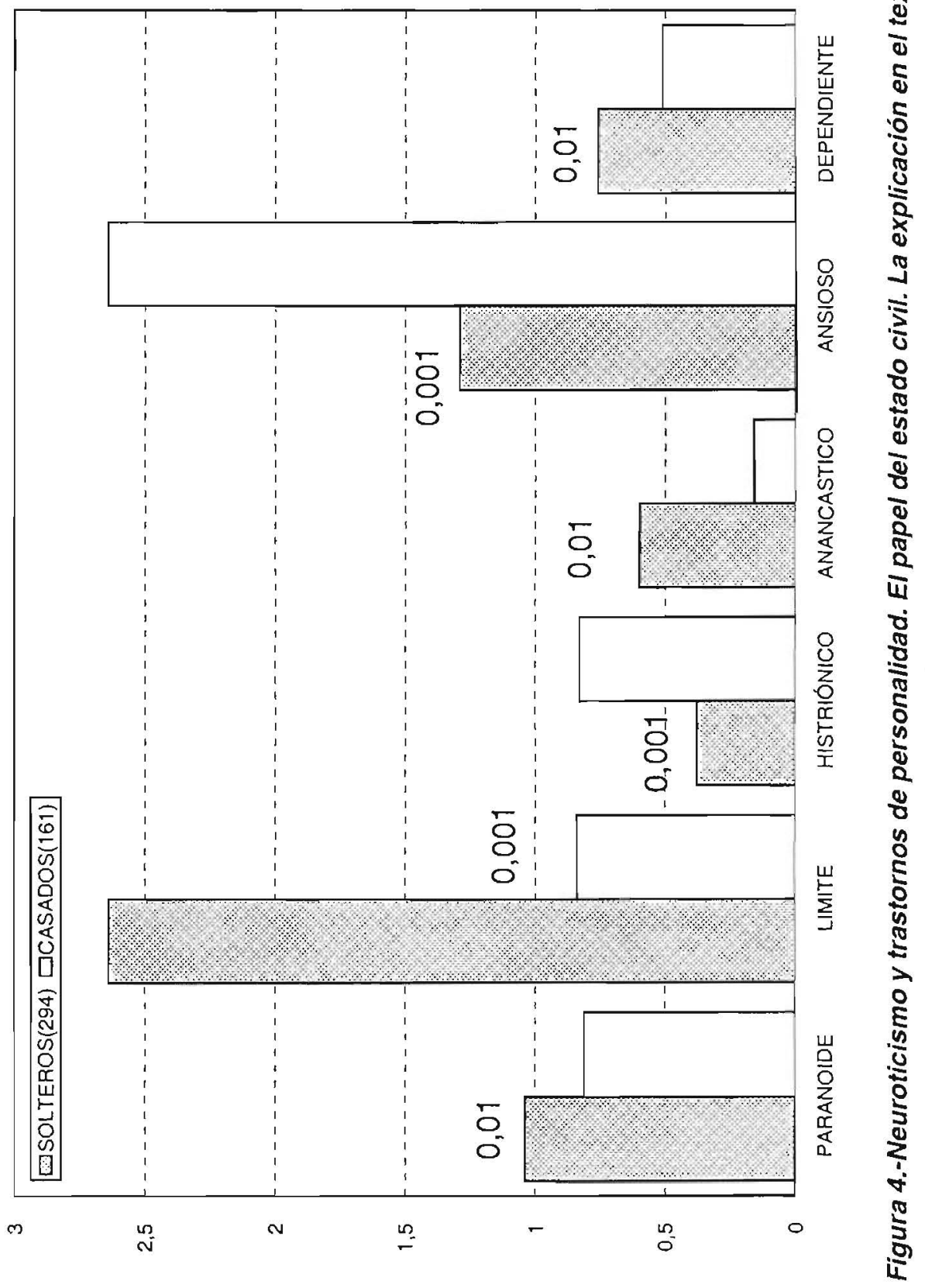


Hay tres trastornos que no presentan diferencias estadísticamente significativas: esquizoide, disocial e impulsivo. En estos tres trastornos no desempeña papel alguno el estado civil. En los otros seis se encuentran diferencias estadísticamente significativas y pueden detectarse dos patrones claros.

En un patrón, la relación es significativamente más intensa en los solteros que en los casados: paranoide, límite, anancástico y dependiente. Esta diferencia debería subrayarse especialmente en el caso del trastorno límite de personalidad.

En el otro patrón sucede justo lo contrario. Los casados presentan una relación más intensa con neuroticismo que los solteros y esto se da en los trastornos histriónico y ansioso.

La razón teórica de estos resultados es desconocida para los autores, aunque sugieren que el neuroticismo-ansiedad resulta más relevante en unos casos que en otros, incluso dentro del mismo trastomo, en función de los criterios de identificación que se utilizan usualmente en la psicologia diferencial. $Y$, de entre todos los tipos de trastornos, el límite parece que presenta patrones muy distintos con neuroticismo $y$, a partir de aquí, el papel que el neuroticismo desempeña en el trastorno límite sea distinto $y$, por ello, el tratamiento que se haga de esta dimensión en los paquetes terapéuticos.

\section{3.d. El papel diferencial del nivel profesional en la relación entre neuroticlsmo y trastornos de personalldad}

Siguiendo el procedimiento descrito de transformación de correlaciones a puntuaciones " $\mathrm{z}$ " y posterior comparación de estas puntuaciones, se han tomado en consideración los siguientes grupos: amas de casa (38), identificadas por no tener horario fijo y una jornada laboral en exceso larga; trabajadores manuales no cualificados (61) tales como peones albañiles; trabajadores manuales cualificados (213) como ebanistas, carpinteros, oficiales de la construcción o electricistas; administrativos y profesiones liberales (64) como empleados de banca, funcionarios y abogados; y estudiantes universitarios (42). Los resultados en forma de representaciones gráficas se encuentran recogidos en las figuras $5 \mathrm{a}, \mathrm{b}$ y $\mathrm{c}$. En las dos primeras figuras se encuentran mezclados. En la tercera los perfiles son claros. 


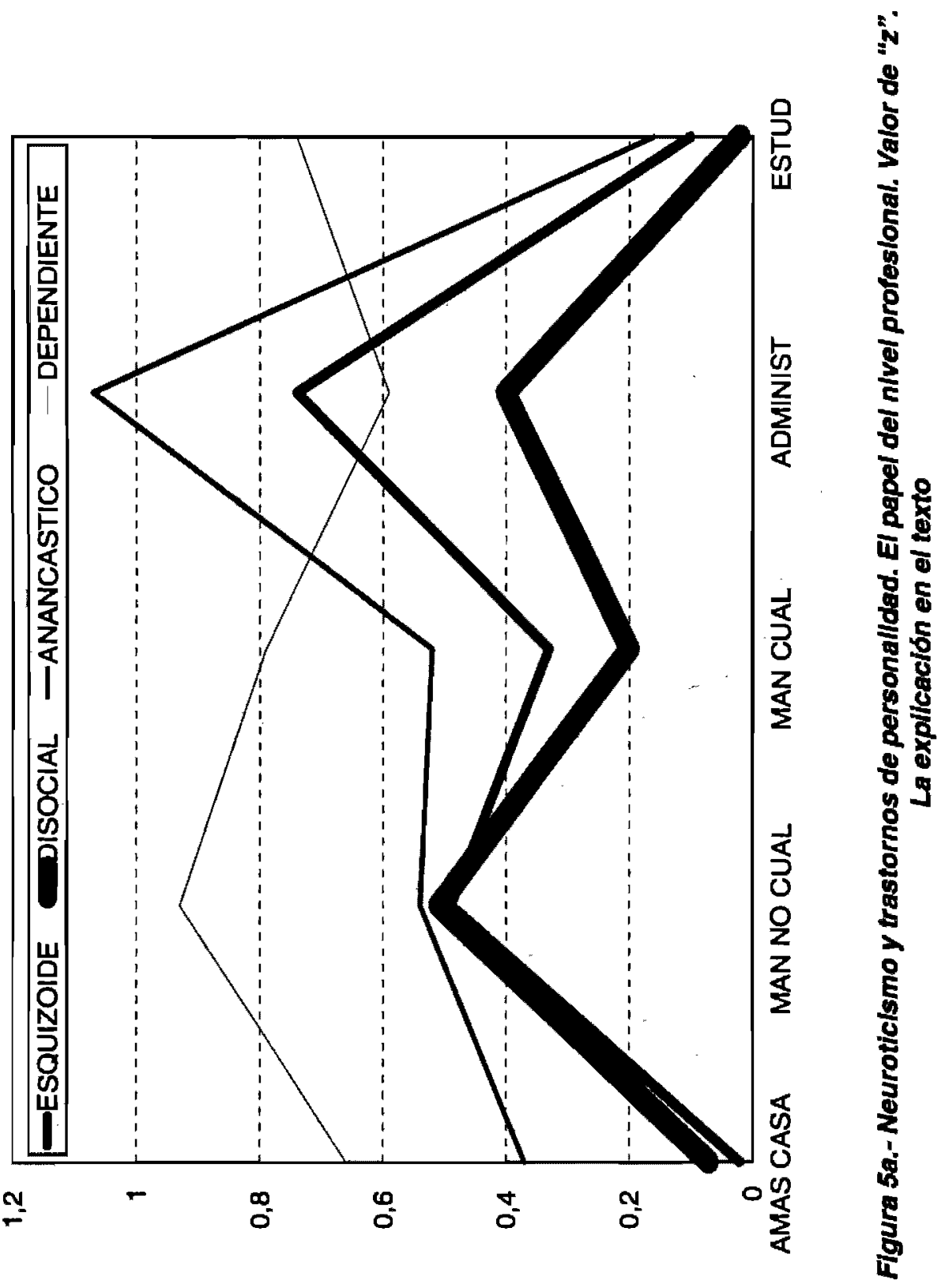




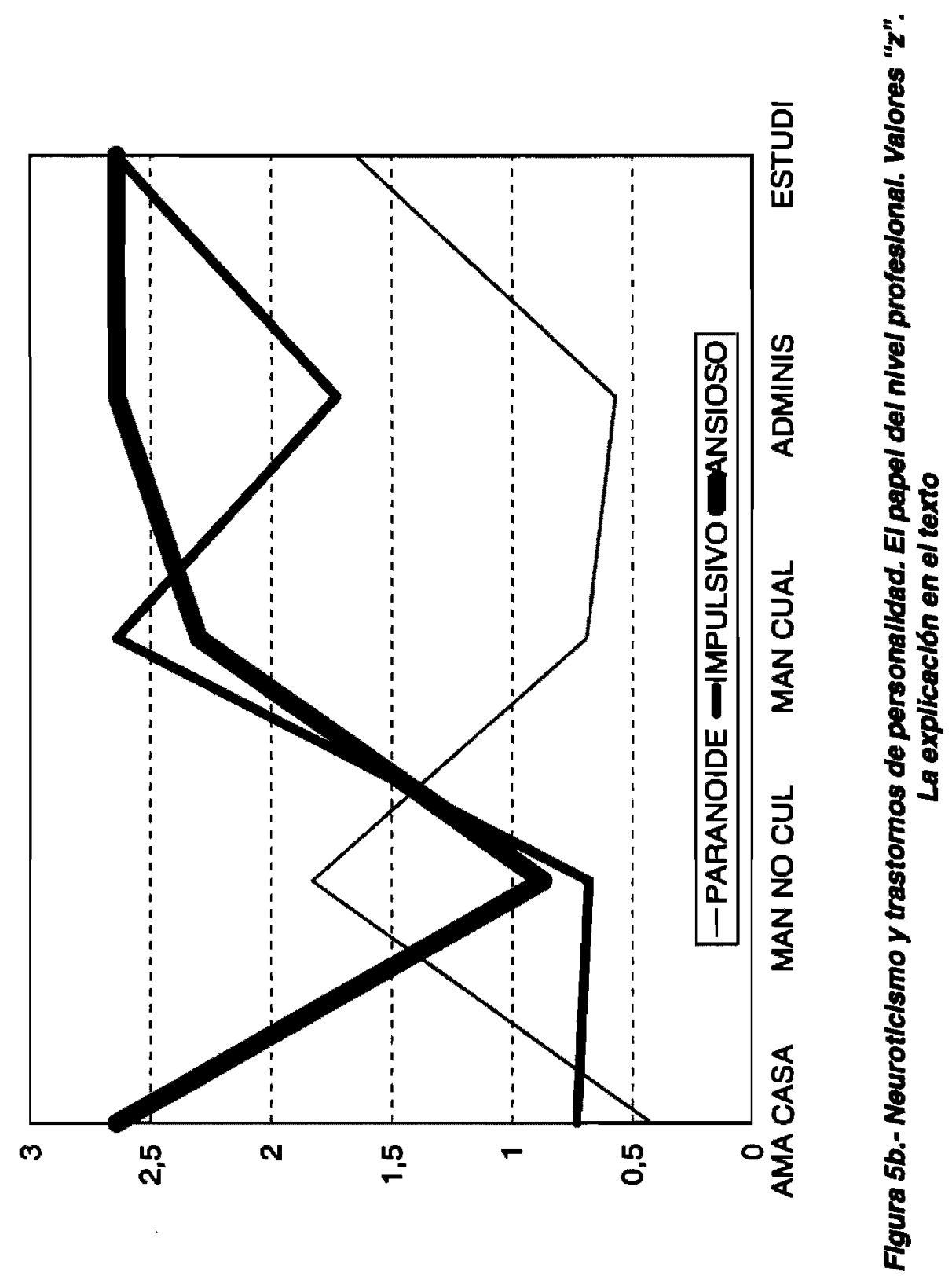




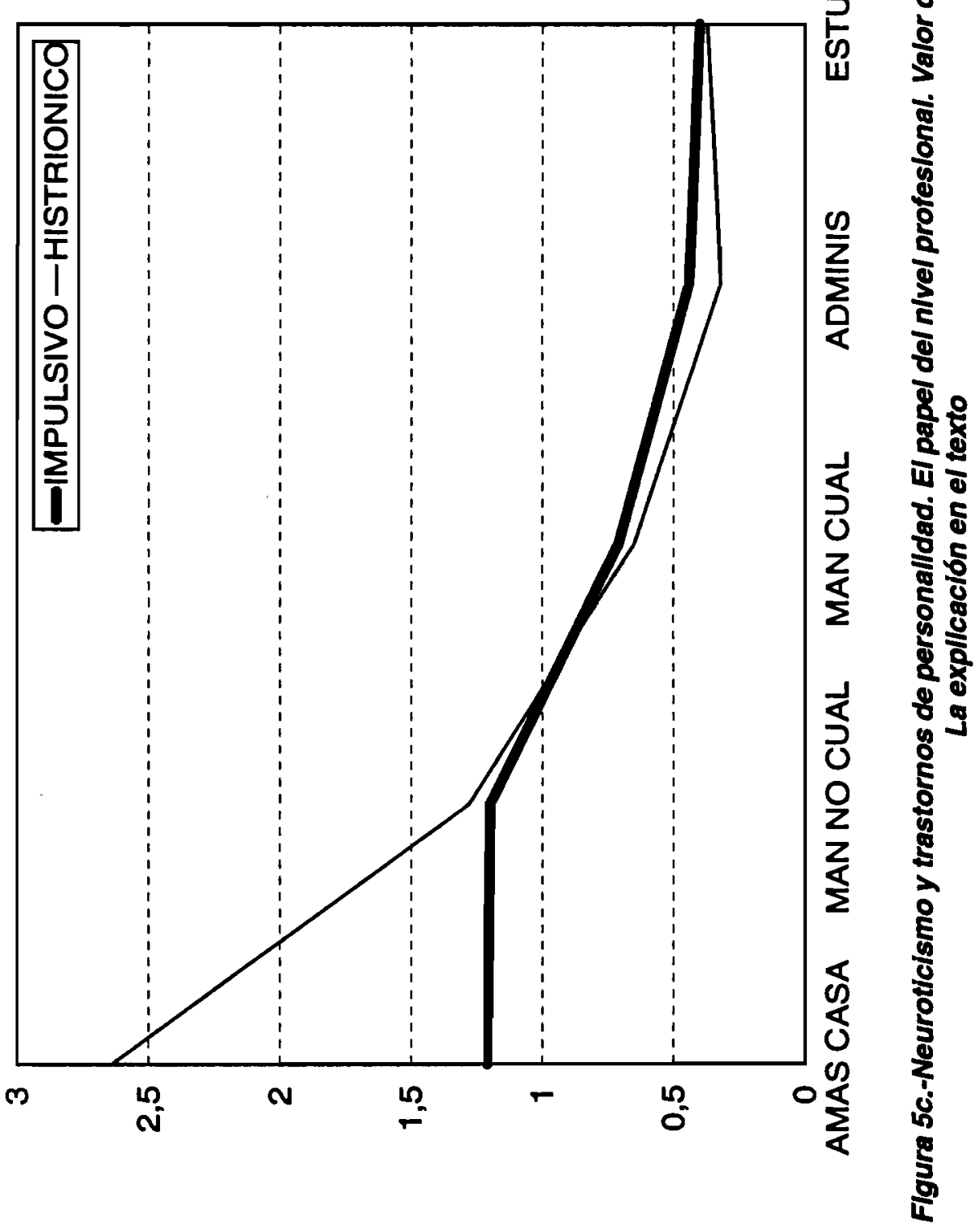


En la figura $5 a$ se encuentran los resultados de las puntuaciones " $\mathrm{z}$ " correspondientes a esquizoide, disocial, anancástico y dependiente. Una carácterística común en todos ellos es que el rango de variabilidad de las puntuaciones " $\mathrm{z}$ " (correlatos de los coeficientes de correlación) es relativamente pequeña. En todos ellos, se observa una "punta" de coeficientes de correlación entre trastornos y neuroticismo y ésta punta es distinta para distintos trastornos: en el trastorno disocial la oscilación es pequeña y las diferencias tienden a localizarse en las comparaciones de amas de casa frente a obreros manuales no cualificados y de éstos frente a estudiantes universitarios. Este mismo perfil, aunque con menor variabilidad se da en el trastorno dependiente.

En el trastorno esquizoide, la punta de mayor relación se da en el grupo de administrativos y profesiones liberales y las diferencias, asimismo se dan entre este grupo y amas de casa por una parte y estudiantes universitarios por otra. El perfil es similar en el trastorno esquizoide. De hecho, tanto en el esquizoide como en el disocial y dependiente podrian detectarse "dos" crestas, correspondientes a trabajadores manuales no cualificados y a administrativos-profesiones liberales. Hay que recordar que el rango de variación es más bien pequeño.

En la figura $5 \mathrm{~b}$ se encuentran los resultados correspondientes al trastorno paranoide, impulsivo y ansioso. El rango de variación es, en estos tres casos mayor que en los que se encontraban en la figura 5a. En el trastorno esquizoide el perfil todavía es similar al que se ha presentado en la figura anterior si bien se observa una relevancia mayor (una segunda punta) en el grupo de estudiantes universitarios. Esta "subida" se encuentra asimismo en los otros dos trastornos (impulsivo y ansioso): la tendencia en el trastorno ansioso es de un compromiso grande en amas de casa, trabajadores manuales cualificados, administrativos/profesiones liberales y estudiantes universitarios. Esta "subida" de las amas de casa en el trastorno ansioso desaparece en los otros dos y, a partir de aquí, el perfil es similar en ellos: incremento desde trabajadores manuales no cualificados a estudiantes universitarios. Y las diferencias son estadísticamente significativas respecto a las amas de casa en unos casos y a los trabajadores no cualificados, en otros. 
En definitiva: parece que el papel de neuroticismo en los trastornos impulsivo y ansioso tiende a ser mayor a mayor cualificación profesional y parece mayor en las profesiones de "cuello blanco" frente a las de "cuello azul". El compromiso de ansiedad con el trastorno ansioso es tan alto como en las profesiones muy cualificadas cuando se estudia a las amas de casa y en el grupo de profesiones manuales no cualificadas parece que la ansiedad es relevante cuando se trate de trastomos paranoides de personalidad.

En la figura $5 \mathrm{c}$ se presentan dos perfiles bastante similares que se refieren a los trastornos impulsivo y al histriónico. El compromiso de neuroticismo con el trastorno histriónico es muy alto en el grupo de amas de casa de manera que parecen cubrir un espectro funcional similar. $Y$ este compromiso tiende a disminuir en los trabajadores no cualificados, después es menos intenso con los de trabajo cualificado y este bloque de "cuello azul" presenta compromisos significativamente mayores que en el caso de administrativos y estudiantes universitarios (en estos dos últimos grupos, no hay variación en cuanto a relación de neuroticismo con los trastornos impulsivo e histriónico y el peso de neuroticismo es pequeño. El perfil correspondiente al trastorno impulsivo es idéntico al comentado hasta aquí excepto en el caso de que amas de casa y trabajadores manuales no cualificados se presentan con un compromiso similar.

\section{CONCLUSIONES Y DISCUSIÓN}

En el planteamiento de este trabajo se asume un modelo dimensional en el estudio de los trastornos de personalidad. Se asume, además, que los trastornos de personalidad son multidimensionales más que unidimensionales (en general) y que, en la medida en que en la definición de trastornos de personalidad se habla de rasgos de personalidad como elementos sustantivos y constitutivos de los mismos, deberian poderse aislar compromisos entre rasgos de personalidad y trastornos de personalidad. Teóricamente, además, la aparición de estos trastornos de personalidad coincide con una manera de estudiar la psicopatología en la que se eliminan conceptos con mucha carga histórica como histeria y neurosis, entidades 
un tanto difusas pero que recogian una gran variedad de casos en los que la curación no era tácil y además, una parte de ellos eran, además, muy difíciles de manejar. En todos ellos se encontraba como marcador la "ansiedad", aunque ésta tenía connotaciones un tanto distintas.

Un primer resultado se refiere a la obtención de relaciones importantes entre neuroticismo y trastornos de personalidad, cuando se analizan los datos desde una determinada perspectiva (eliminación de varianza de error a base de correcciones pro atenuación), lo que implica que se muestra un camino de estudio en el que la psicología de la personalidad y los trastornos de personalidad se pueden acercar con garantías cientificas.

Un segundo gran resultado alcanzado es que, en determinadas condiciones y trastornos el neuroticismo aparece como el principal determinante de ciertos trastornos de personalidad (ansioso, límite), prácticamente identificados las escalas de trastornos y la dimensión de neuroticismo. El trastorno esquizoide y el disocial, sin embargo, parecen ser determinados, tan solo en parte, por el neuroticismo. Desde aquí la posible sugerencia de reconducir parte de la investigación y de la práctica, en una vuelta a procedimientos eficaces para el tratamiento de la ansiedad. Cuando a ésta se une la depresión, asimismo debería reconducirse.

Un tercer punto de interés se refiere a la existencia de distintos patrones en la relación de neuroticismo con trastornos de personalidad en función de la acción de unos moduladores que se utilizan en la investigación en psicología diferencial. Hemos presentado resultados que sugieren que el papel del neuroticismo en distintos trastomos de personalidad es distinto en función de la edad, estado civil, género y nivel profesional (en unos casos, relaciones lineales $y$ en otros no), que no todos los trastornos parecen igualmente sensibles a la modificación de las relaciones y que en muchas de estas moditicaciones pueden sugerirse insistencia en unos u otros componentes a la hora de elegir procedimientos terapéuticos. El panorama que se desprende de estos resultados modificaría los codeterminantes de los éxitos terapéuticos así como la elección de unos $\mathrm{u}$ otros procedimientos de intervención. 
En cuarto lugar, somos consciente de fuertes limitaciones que poseen estos resultados: se trata de una muestra de personas "normales" lo que dificulta la generalización de resultados a muestras clínicas. Más aún, cuando un tema central en los distintos resultados se centra en la obtención de modificaciones de la relación entre escalas de trastornos y neuroticismo en función de ciertas modulaciones. En muchos sentidos este trabajo puede representar una fuente de hipótesis que se deberian contrastar con otras muestras y condiciones experimentales. El sentido seminal y de sugerencia que poseen estos resultados no debe ignorar que se trata además de unas ideas, de resultados empiricos novedosos y que pueden servir como plataforma de discusión y de arranque de investigaciones.

\section{BIBLIOGRAFIA}

American Psychlatric Association (APA) (2000).- Diagnostic and statistical manual of mental disorders (DSM-IV-TR),Washington, DC, American Psychological Association.

Costeilo, C. G. (1996).- The advantages of focusing on the personality characteristics of the personality disordered. En Costello, C. G. (ed).Personality characteristics of the personality disordered, New York, John Wiley \& Sons.

Deary, J. A., Peter, A., Austln, E. \& Glbson, G. (1998).- Personality traits and personality disorders, British Joumal of Psychology,89, 647-661.

Eysenck, H. J., \& Eysenck, S. B. G. (1964).- Manual of the Eysenck Personality Inventory, London, University of London Press.

Lenzenberger, M. F., Loranger, A. W., Korfine, L. \& Neff, C. (1997).Detecting personality disorders in a nonclinical population. Application of a 2-stage procedure for case identification, Archives of General Psychiatry, $55,345-351$.

Llvesley, W. J., Jang, K. L. \& Vernon, P. A. (1988).- Phenotypic and genetic structure of traits delineating personality disorder, Archives of General Psychiatric, 55, 941-948.

Loranger, S. W. (1988).- Personality Disorder Examination (PDE) Manual, Yonkers, DV: Communications.

Loranger, S. W., Janca, A. \& Sartorlus,S (1997).- Assessment and diagnosis of personality disorders. 
Miguel, A. de \& Pelechano, V. (2000).- Estructura de personalidad y trastornos de personalidad: correlatos de personalidad de los trastornos de personalidad definidos por clasificaciones de consenso, Psicologemas, 14, 3-208.

Organización Mundlal de la Salud (OMS) (1992).- Clasificación Internacional de las Enfermedades, (10 edición), (ClE-10), Madrid, Meditor.

Pelechano, V. (1993).- Psicologia de la personalidad. Un acercamiento histórico-conceptual, Valencia, Promolibro.

Pelechano, V. (1996).- Una introducción al modelo de parámetros en psicologia. En V. Pelechano (coord).- Psicología de la personalidad.I.Teorías, Barcelona, Ariel.

Pelechano, V. (2000).- Psicologra sistemática de la personalidad, BarceIona, Arie.

Pelechano, V. \& Servando, M. A. (2004).- ¿Qué es la personalidad?,Madrid, Biblioteca Nueva. 